\title{
Habituation of gastric function suppression in monkeys after repeated free-operant avoidance sessions
}

\author{
ANDRÉ DUBOIS \\ Department of Internal Medicine, Uniformed Services University of the Health Sciences \\ Bethesda, Maryland 20014
}

and

\author{
BENJAMIN H. NATELSON \\ Veterans Administration Hospital and Department of Neurosciences, New Jersey Medical School \\ East Orange, New Jersey 07018
}

\begin{abstract}
Gastric fluid and hydrogen ion secretory rates as well as concurrent fractional emptying rates were determined in monkeys subjected to repeated sessions of free operant avoidance. During the first avoidance session, fractional emptying rate and fluid and hydrogen ion secretory rates were all reduced as compared to preavoidance control rates. By the time the monkeys were performing avoidance stably, fluid and hydrogen ion secretory rates had returned to control levels while fractional emptying tended to return to control levels. Our interpretation that this represented habituation of the stomach to the stressor was buttressed by another experiment in which response-contingent shock was superimposed upon the baseline avoidance schedule. Gastric fractional emptying and fluid and hydrogen ion secretory rates remained at control levels despite the increase in shock frequency related to this new paradigm.
\end{abstract}

Repeated sessions of free-operant avoidance have been reported to produce, in monkeys, chronic peptic ulcers (Brady, Porter, Conrad, \& Mason, 1958), a disease associated with hypersecretion of acid in man (Brooks, 1974). In follow-up studies, Polish, Brady, Mason, Thach, and Niemeck (1962) periodically sampled gastric juice for acid concentration during the first 6-h avoidance session and then between 6-h avoidance sessions on a weekly basis. Their most striking finding was a dearth of free acid during the first session. Gastric juice was not assayed during subsequent experimental sessions, so it is not known whether hypersecretion of acid occurred in the chronic

These experiments were performed in the Department of Experimental Psychology of the Walter Reed Institute of Research. Gastric samples were assayed for kinetic data in the Section of Gastroenterology, Digestive Diseases Branch, National Institute of Arthritis, Metabolism and Digestive Diseases. Preparation of this report was supported by VA medical research funds to B.H.N. In conducting the research described in this report, the "Guide for Laboratory Animal Facilities and Care," as promulgated by the Committee on the Revision for Laboratory Animal Facilities and Care of the Institute of Laboratory Resources, National Academy of Sciences-National Research Council, was followed. The authors acknowledge the technical help of T. Queen and the secretarial skills of L. Fallone. Requests for reprints should be addressed to: Benjamin H. Natelson, M.D., Department of Neurosciences, New Jersey Medical School, 88 Ross Street, East Orange, New Jersey 07018. state. When gastric juice was tested between experimental sessions, acid concentration returned to baseline levels and was occasionally increased. Since rate of acid secretion was not measured, it is unclear whether these changes in acidity were due to changes in acid or in fluid secretion rates. Smith (1967) considers these changes difficult to evaluate because of the lack of accurate volume measurements inherent in the sampling technique used by Polish et al.

In later experiments on rats, Paré, who continuously collected gastric juice prior to and during shock avoidance sessions, reported that gastric volume remained low during repeated avoidance sessions. Similarly, acid secretion rate (computed by us from Paré's data) was suppressed over repeated avoidance sessions (1972).

In trying to compare these two sets of experiments, we could not determine whether the differences were methodological or related to actual differences across the species. Evidence for a species difference is strong for chronic peptic ulcer disease: Peptic ulcers (chronic deep lesions occurring in nonacid-producing gastric antrum and/or duodenum (Brooks, 1974; Oi, Toriumi, Miho, \& Kijima, 1971) do not spontaneously occur in rats, but do in monkeys (Ruch, 1959, pp. 255-256). Thus, these experiments were conducted to evaluate the effect of repeated sessions of free-operant avoidance (Sidman, 1953) on monkey gastric function. 


\section{METHOD}

\section{Animals}

Four adult male rhesus monkeys (Macacca mulatta), weighing 6-8 kg, were maintained in primate living chairs, individually enclosed in sound-attenuated booths lit $12 \mathrm{~h}$ a day. Food and water were available from 3 to 5 p.m.

\section{Method of Determining Gastric Function}

Monkeys were surgicaly prepared with esophagostomies by an aseptic technique described elsewhere (Dubois, Watanabe, \& Kopin, 1973). During the latter part of the 2- to 3-week postoperative recovery period, several preparatory sessions were held to accustom animals to accept a $12 \mathrm{fr}$. double-lumen Salem sump tube, inserted through the esophagostomy into the stomach, and hand restraints to prevent them from removing the tube. Rates of gastric emptying and secretion were determined as detailed elsewhere (Dubois, Natelson, Van Eerdewegh, \& Gardner, 1977); a brief description of the method follows. It is based on the use of a dye marker, phenosulfonephthalein (PSP), which is not significantly absorbed, secreted, or degraded by the stomach (Ivey \& Schedl, 1970).

Aliquots of gastric juice were collected before and after instillation of a volume-for-volume replacement of a PSP test solution $(280 \mathrm{mg} / \mathrm{liter})$. These samplings were repeated at $10-\mathrm{min}$ intervals during a 30 -min fasting period, 5 and $10 \mathrm{~min}$ after infusion of a $100-\mathrm{ml}$ water test "meal" ( $40 \mathrm{mg}$ PSP/liter) and again at 10-min intervals during the subsequent $40 \mathrm{~min}$. After centrifugation, concentration of PSP was measured spectrophotometrically in the clear supernatant of all samples of gastric juice, in the test solution and in the test meal (Gilford Microsample Spectrophotometer, Oberlin, Ohio), after appropriate dilution with $.25 \% \mathrm{Na}_{3} \mathrm{PO}_{4}(\mathrm{pH} \mathrm{10})$. Hydrogen ion concentration was determined in each sample by electrometric titration to $\mathrm{pH} 7.4$ with $.01 \mathrm{~N} \mathrm{NaOH}$ (Titration assembly, Radiometer, Copenhagen, Denmark). Using the Fick principle, the amounts of fluid, PSP, and hydrogen ion present in the gastric contents were calculated at the time of each dilution procedure. Since PSP is unaffected by the stomach, the rate of decline of its intragastric contents is the rate of emptying for PSP. This rate, divided by the intragastric amount of phenol red, is the fractional rate of emptying (FRE).

The same FRE also applies to any component of the gastric contents which is homogenously mixed with PSP, such as water, hydrogen ion, etc. The rate of fluid secretion is then determined from the difference between the rate of change of the intragastric fluid volume and the rate of fluid emptying. Hydrogen ion secretory rate is calculated similarly (Dubois et al., 1977).

\section{Experimental Design}

From two to four control studies of gastric function were performed on each subject. Following control sessions, animals were shaped by successive approximations to press a lever to escape experimenter-controlled electric shocks, delivered across feet and legs by a constant current shocker (Techserv; Model 113-08; Beltsville, Maryland). The criterion for adequate escape performance was that the monkey consistently escaped randomly programmed trains of pulsed shock, initiated on the average of $1.5 / \mathrm{h}$ during alternating $6-\mathrm{h}$ periods. On the day after attaining this criterion, the animals were switched to a free operant avoidance schedule with an RS interval of $20 \mathrm{sec}$ and an SS interval of $1 \mathrm{sec}$ (Sidman, 1953); shocks were $7.2 \mathrm{~mA}$ in intensity and $200-400 \mathrm{msec}$ in duration. Avoidance sessions were not signaled to the animal and were held from 3 to 9 p.m. and from 3 to 9 a.m.; the first such session began at about 2 p.m., and a study of gastric function was begun about $\mathbf{3 0}$ min later. A final study was done when a monkey's response and shock rates showed no consistent changes over 3 days: This criterion was reached in 1-3 weeks, and shock rates during this study averaged 2, 3, 4 , and 75 shocks $/ \mathrm{h}$ for the four monkeys.
Following this study, the monkeys continued to perform shock avoidance for an additional 1-2 weeks. Then a responsecontingent shock schedule was superimposed upon the baseline avoidance schedule. This schedule specified that $5 \%$ of all avoidance responses, gated randomly by a probability generator, would be immediately followed by a 200 -msec, 5-mA shock. Gastric function was studied during the first such 6-h session in the four monkeys, but results were technically satisfactory in only three animals.

Values obtained from individual animals for each interval before and after the meal during the first and last avoidance sessions were used to determine the means given in Figures 1 to 4. Repeated control studies were averaged to provide one set of control data per monkey. Fasting values were averaged to provide one value for each of the three conditions studied. We evaluated the statistical significance of changes in each function (e.g., fractional emptying, fluid secretion, etc.), using a two-factor analysis of variance (session type and time within sessions) with repeated measures and subsequent Tukey tests (Kirk, 1968, pp. 110-114).

\section{RESULTS}

During control sessions, administration of the water meal produced a large, immediate increase in intragastric volume. Volume then decreased, rapidly at first and then more slowly toward the end of the 50-min time period studied (Figure 1). Intragastric volume was significantly increased from control both during the first and last sessions of freeoperant avoidance $[q(3,6)=6.74$ and 4.49 , respectively; $p<.05$, Figure 1]. Volumes found during these two avoidance sessions did not differ significantly from one another.

These changes in intragastric volume reflect simultaneous changes in rates of emptying and fluid secretion. During control sessions, fractional rate of emptying increased over threefold during the infusion

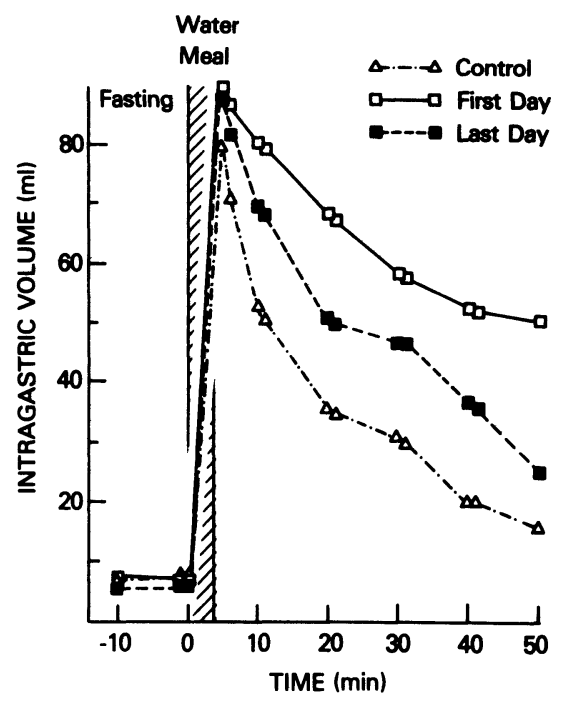

Figure 1. Intragastric volume during fasting and following a 100-ml water meal in chair-adapted rhesus monkeys. Each point illustrates the mean of values obtained during control sessions and on the first and last days of 6-h shock-avoidance sessions. 


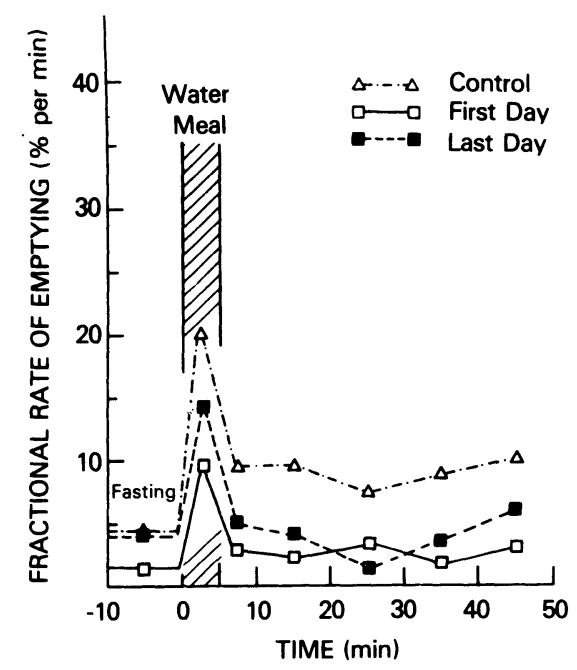

Figure 2. Fractional rate of gastric emptying during fasting and following a $100-\mathrm{ml}$ water meal. Each point represents the mean of values obtained during control sessions and on the first and last days of shock avoidance.

of the water meal. As depicted in Figure 2, during both the first and last avoidance sessions, FRE was significantly decreased from control levels $[q(3,6)$ $=8.52$ and $5.94 ; \mathrm{p}<.01$ and .05 , respectively]. FRE during these avoidance sessions did not differ when one was compared to the other. A significant inverse correlation was found during the last session between average shock density and average FRE after the meal $(\mathrm{r}=-.965, \mathrm{n}=4$, df $=2, \mathrm{p}<.05)$.

During control sessions, fluid secretion tended to parallel FRE by showing the major increase during the infusion of the water meal with a subsequent return toward fasting levels. This pattern was significantly decreased during the first shock avoidance session $[q(3,6)=5.98, p<.05$, Figure 3$]$; rate of fluid secretion had returned to control levels by the last avoidance session. Although fluid secretion was diminished during the first avoidance session, a simultaneous decrease of FRE was responsible for the greater postprandial intragastric volume seen during that session (Figure 1).

During control sessions, infusion of the water meal produced increases in rate of hydrogen ion secretion which peaked by $30 \mathrm{~min}$ after the start of the infusion. Rate of hydrogen ion secretion was significantly decreased from control levels during the first avoidance session $[q(3,10)=4.12 ; p<.05]$ but not during the last (Figure 4).

Relative to the rate at which they worked during the last avoidance session [average responses $/ \mathrm{min}=$ $29 \pm 10$ (SE)], all four monkeys responded faster during the first session when response-contingent shock was superimposed on the avoidance schedule $[56 \pm 15, \mathrm{t}(3)=4.06, \mathrm{p}<.05]$. As a consequence of this increased rate, the four monkeys self- inflicted 68, 130, 179, and 287 shocks, respectively, per hour.

Despite the large increase in shock frequency noted here, intragastric volume, its component subunits-

\section{FLUID SECRETION}

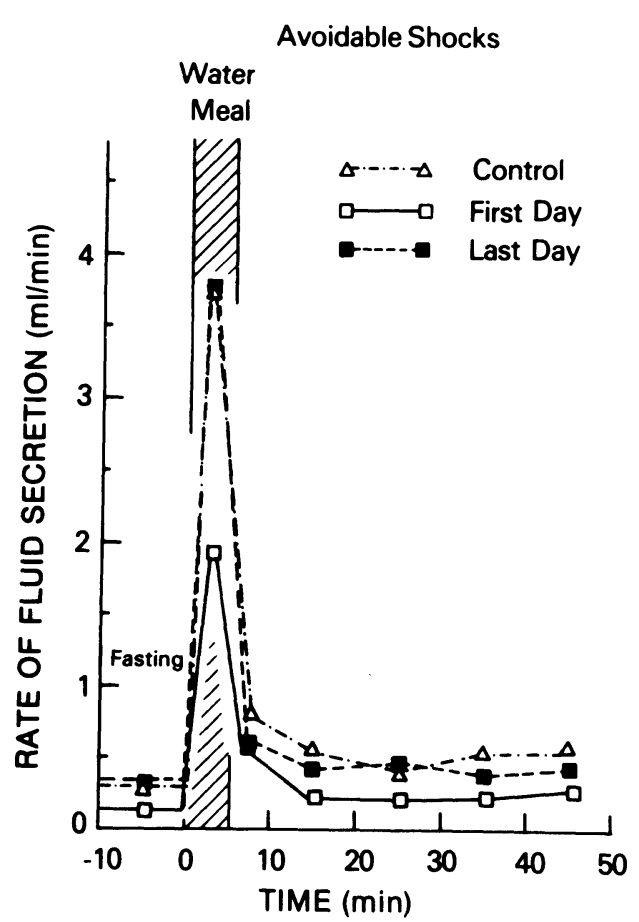

Figure 3. Rate of fluid secretion during fasting and following a 100-ml water meal. Each point illustrates the mean of values obtained during control sessions and on the first and last days of 6-h shock-avoidance sessions.

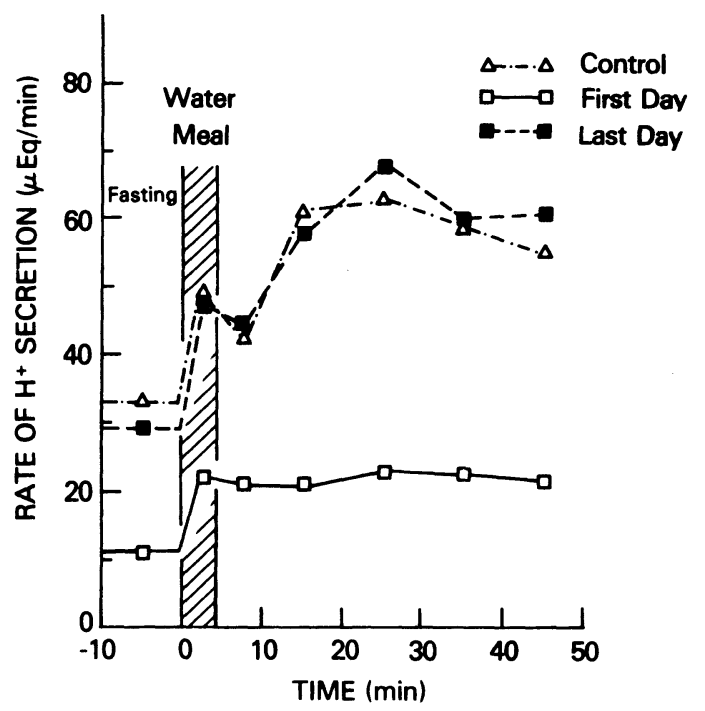

Figure 4. Rate of hydrogen ion secretion during fasting and following a $100-\mathrm{ml}$ water meal. Each point illustrates the mean values obtained during control sessions and on the first and last days of shock avoidance. 
FRE and fluid secretion rate-and hydrogen ion secretion rate were not significantly different from data found during both the control session or the last avoidance session.

\section{DISCUSSION}

The effects of acute stress on gastric motility and secretion have been known for nearly a century: Both secretion (Bickel \& Sasaki, 1905) and motility (Cannon, 1898) are inhibited. The unresolved question is what happens to gastric function when stressful sessions are repeatedly presented to animals. This is especially important during repeated sessions of free-operant avoidance, a procedure which has been reported to produce duodenal ulcers in monkeys (Brady et al., 1958). Our data answer this question in the monkey: By the time monkeys are stably performing free-operant avoidance, gastric secretion has returned to control levels and fractional rate of gastric emptying, though still suppressed, shows a trend toward return to control rates. Polish et al. (1962) intermittently sampled gastric juice for acid concentration between sessions in monkeys performing free-operant avoidance for 1 month. In contrast to the uniform decrease in acidity in the first session, they noted that inhibition was not uniformly observed and that acid concentrations were very variable, with a transient hyperacidity seen during the 2 nd week of conditioning. Our data, plus those of Polish et al. (1962), rule out sustained hyperacidity as the etiologic factor responsible for the ulcers produced by Brady et al. (1958). A question for further research is whether focal gastroduodenal disease appears in the stressed monkey, because this area of the gut has an increased vulnerability to normal amounts of acid (Natelson, Dubois, \& Sodetz, 1977).

A number of different possibilities can explain the return of gastric function to normal after repeated exposure to sessions of free-operant avoidance. The simplest explanation is that the monkeys learned to perform the avoidance task and thus changed the experimental situation by eliminating much of the nociceptive stimulation. That this is not the case is indicated by the fact that gastric function remained at control levels even during a session when large numbers of response-contingent shocks were superimposed on the baseline avoidance session. The other major explanation, and the one we favor, relates to the phenomenon known as "habituation," operationally defined to be "response decrement as a result of repeated stimulation (Harris, 1943, p. 385). To be sure habituation is occurring, one must eliminate the possibilities of peripheral mechanisms such as "receptor adaptation" and "effector fatigue" (Thompson \& Spencer, 1966). A common way of doing this is to see whether the presentation of another strong stimulus results in recovery of the habituated response (dishabituation) (Thompson \& Spencer, 1966). Our superimposition of responsecontingent shock was directed to this.

During the study period, monkeys delivered a a rather high density of strong shocks to themselves. Since their operant response doubled during this session, it seems obvious that the animals had perceived a change in stimulus conditions. Thus peripheral sensory receptors and central processing of the consequences of the stimuli remained intact. Yet, gastric function remained normal. Although this may mean that a peripheral mechanism is operative, it also may mean that the response-contingent shock schedule was not sufficiently different from the original avoidance paradigm to produce dishabituation. One of the characteristics of habituation to a particular stimulus is that the habituated response often generalizes to other stimuli which resemble the original stimulus in some fashion (Thompson \& Spencer, 1966).

Having not shown dishabituation, our interpretation of loss of suppression of gastric function as an example of habituation must remain tentative. However, data from our own earlier work on adrenal glucocorticoids (Natelson, Krasnegor, \& Holaday, 1976) makes us confident that the reason for the loss of responsiveness reported here is central and not peripheral. In that experiment, we demonstrated that the large increase in plasma cortisol seen during the first 6-h session of shock avoidance disappeared in later sessions and could not be restored by the superimposition of response-independent shocks. However, when the chaired monkeys were then removed from their booths and placed in a noisy hallway, dishabituation occurred, and mean plasma cortisol levels increased by $50 \%$.

The degree of loss of visceral responsivity shown here may relate to the fact that these monkeys received many shocks early in their training. This is suggested by the work of Thompson and Spencer (1966), who have noted that frequency of stimulus is an important physical variable in producing habituation. The other important alternative is that it was the psychological contingencies inherent in allowing a coping response that produced this effect (Weiss, 1971). Further experiments, using yoked controls, will be needed to sort this problem out.

The former reason may explain why gastric function returned to normal in these monkeys performing Sidman avoidance but remained suppressed in rats (Paré, 1972). In his study, Paré first taught rats to perform free-operant shock avoidance $(R S=24$; $\mathrm{SS}=6$ ); he then measured gastric secretion and acid concentration during six 3 -h sessions, in which the rats avoided shock in the 2nd hour; sessions 
were separated by at least 3 days. Even in the sixth session, secretion was decreased and acid concentration increased. In our study, avoidance sessions were $12 \mathrm{~h}$ daily, the SS was $1 \mathrm{sec}$, and studies were done at least 1 week after the start of the experiment: thus, our monkeys had greater exposure to the overall aversive paradigm and probably received more shocks each day than did the rats. This interpretation requires experimental confirmation.

A less compelling reason for the differences might relate to interspecies variability in habituating to shock avoidance. Both avoidance-induced suppression of gastric function and avoidance-induced increases in adrenocorticoids return to control levels in monkeys (this study and Natelson et al., 1976) but not in rats (Coover, Ursin, \& Levine, 1973; Paré, 1972). However, we believe that more complete habituation of these visceral changes might have been seen in rats if the avoidance sessions had been longer and/or if the sessions had been held in the rat's home environment rather than in a novel experimental chamber.

The individual components comprising gastric visceral function did not return to baseline levels at the same rate; thus, fluid and hydrogen ion secretory rates had returned to control levels by the time monkeys demonstrated stable avoidance behavior, but fractional rate of emptying remained suppressed at that time, It, too, finally returned to baseline levels, and like the other variables did not show suppression following the introduction of a punishment paradigm. We have noted this dissociation in previous experiments done 1 day after the stress of laparotomy (Dubois \& Berman, 1973). Normal hydrogen ion secretion and decreased fractional gastric emptying represents an imbalance of gastric function. Such an imbalance during stress could explain why "stress ulcers," unlike chronic peptic ulcers, develop preponderantly in the acid-producing mucosa of the stomach of experimental animals and man (Oi et al., 1971).

\section{REFERENCES}

Bickel, A., \& SASAKI, K. Experimentelle Untersuchungen über den Einfluss von Affekten auf die Magensaftsekretion Deutsche Medizinische Wochenschrift, 1905, 31, 1829-1831.

Brady, J. V., Porter, R. W., Conrad, D. G., \& Mason, J. W. Avoidance behavior and the development of gastroduodenal ulcers. Journal of the Experimental Analysis of Behavior, 1958, 1, 69-72.

Brooks, F. P. Gastroduodenal disease. In F. P. Brooks (Ed.), Gastrointestinal pathophysiology. New York: Oxford Press, 1974.

Cannon, W. B. The movements of the stomach studied by means of the roentgen rays. American Journal of Physiology, 1898, 1, 359-382.

Coover, G. D., Ursin, H., \& Levine, S. Plasma-corticosterone levels during active-avoidance learning in rats. Journal of Comparative and Physiological Psychology, 1973, 82, 170-174.

Dubois, A., \& BERMAN, M. Gastric emptying and secretion: Kinetic analysis and physiological model. In Proceedings of the Fourth International Symposium on Gastrointestinal Motility, 1973, 523-536.

Dubois, A., Natelson, B. H., Van Eerdewegh, P., \& GARDNER, J. D. Gastric emptying and secretion in the rhesus monkey. American Journal of Physiology, 1977, 232, E186-E192.

Dubois, A., Watanabe, A. M., \& Kopin, I. J. Postoperative gastric ileus. American Journal of Digestive Diseases, 1973, 18, $39-42$.

HARRIs, J. D. Habituatory response decrement in the intact organism. Psychological Bulletin, 1943, 40, 385-422.

IVEY, K. J., \& SCHEDL, H. P. Gastric non-absorbable indicators for studies in man. Gastroenterology, 1970, 59, 234-239.

KIRK, R. E. Experimental design: Procedures for the behavioral sciences. Belmont, Calif: Brooks/Cole, 1968.

Natelson, B. H., Dubois, A., \& Sodetz, F. J. Effect of multiplestress procedures on monkey gastroduodenal mucosa, serum gastrin and hydrogen ion kinetics. American Journal of Digestive Diseases, 1977, 22, 888-897.

Natelson, B. H., Krasnegor, N., \& Holaday, J. W. Relations between behavioral arousal and plasma cortisol levels in monkeys performing repeated free-operant avoidance sessions. Journal of Comparative and Physiological Psychology, 1976, 90, 958-969.

Oi, M., Toriumi, T., Miro, O., \& Kijima, M. Location of experimental ulcers as compared with that of human peptic ulcer. In C. F. Pfeiffer (Ed.), Peptic ulcer. Philadelphia: Lippincott, 1971.

PARÉ, W. Conditioning and avoidance responding effects on gastric secretion in the rat with chronic fistula. Journal of Comparative and Physiological Psychology, 1972, 80, 150-162.

Polish, E. Brady, J. W., Mason, J. W. Thach, J. S., \& NIEMECK, W. Gastric contents and the occurrence of duodenal lesions in the rhesus monkey during avoidance behavior. Gastroenterology, 1962, 43, 193-201.

Ruch, T. C. Diseases of laboratory primates. Philadelphia: Saunders, 1959.

SIDMAN, M. Avoidance conditioning with brief shock and no exteroceptive warning signal. Science, 1953, 118, 157-158.

SMITH, G. P. Experimental ulcer and limbic system. In C. Thompson, D. Berkowitz, \& E. Polish (Eds.), The stomach. New York: Grune and Stratton, 1967.

Thompson, R. F., \& Spencer, W. A. Habituation: A model phenomenon for the study of neuronal substrates of behavior. Psychological Reviews, 1966, 173, 16-43.

WEISS, J. M. Effects of coping behavior in different warning signal conditions on stress pathology in rats. Journal of Comparative and Physiological Psychology, 1971, 29, 1-13.

(Received for publication April 11, 1978; revision accepted September $8,1978$. ) 